\title{
Bacterial diversity associated with the tunic of the model chordate Ciona intestinalis
}

\author{
Leah C Blasiak ${ }^{1}$, Stephen H Zinder ${ }^{2}$, Daniel H Buckley ${ }^{3}$ and Russell T Hill ${ }^{1}$ \\ ${ }^{1}$ Institute of Marine and Environmental Technology (IMET), University of Maryland Center for Environmental \\ Science, Baltimore, MD, USA; ${ }^{2}$ Department of Microbiology, Cornell University, Ithaca, NY, USA and \\ ${ }^{3}$ Department of Crop and Soil Sciences, Cornell University, Ithaca, NY, USA
}

\begin{abstract}
The sea squirt Ciona intestinalis is a well-studied model organism in developmental biology, yet little is known about its associated bacterial community. In this study, a combination of 454 pyrosequencing of $16 \mathrm{~S}$ ribosomal RNA genes, catalyzed reporter deposition-fluorescence in situ hybridization and bacterial culture were used to characterize the bacteria living inside and on the exterior coating, or tunic, of $C$. intestinalis adults. The 454 sequencing data set demonstrated that the tunic bacterial community structure is different from that of the surrounding seawater. The observed tunic bacterial consortium contained a shared community of $<10$ abundant bacterial phylotypes across three individuals. Culture experiments yielded four bacterial strains that were also dominant groups in the $\mathbf{4 5 4}$ sequencing data set, including novel representatives of the classes Alphaproteobacteria and Flavobacteria. The relatively simple bacterial community and availability of dominant community members in culture make $C$. intestinalis a promising system in which to investigate functional interactions between host-associated microbiota and the development of host innate immunity.
\end{abstract}

The ISME Journal (2014) 8, 309-320; doi:10.1038/ismej.2013.156; published online 19 September 2013

Subject Category: Microbe-microbe and microbe-host interactions

Keywords: $16 \mathrm{~S}$ rRNA gene; microbiome; tunicate; ascidian; symbiont; CARD-FISH

\section{Introduction}

The tunicate Ciona intestinalis has been studied as a model organism in developmental biology since the late 1800 s, and has recently regained popularity as an experimental model because of advances in sequencing and molecular biology. Tunicates are named for their protective outer coating or tunic, which in $C$. intestinalis consists of a layer of cellulose and mucopolysaccharide exterior to the epidermis (De Leo et al., 1981). Several studies have documented abundant bacteria both in and on the tunic of Ciona (De Leo and Patricolo, 1980; De Leo et al., 1981; Di Bella et al., 2011). However, the phylogeny and ecological role of the tunic bacteria remain completely unknown. This host-associated bacterial community is of special interest given the tractability of Ciona as an experimental system.

Several factors have contributed to the resurgence of Ciona as a model organism, not least the availability of both the $C$. intestinalis and the related

Correspondence: RT Hill, Institute of Marine and Environmental Technology, University of Maryland Center for Environmental Science, Columbus Center Suite 236, 701 East Pratt Street, Baltimore, MD 21202, USA.

E-mail: hill@umces.edu

Received 22 February 2013; revised 7 May 2013; accepted 29 July 2013; published online 19 September 2013
C. saviygni genome sequences (Dehal et al., 2002; Small et al., 2007). The Ciona genomes are the smallest sequenced chordate genomes at $150-170 \mathrm{Mb}$ in length and encode $\sim 16000$ genes. In addition, molecular and genetic methods are well established in Ciona, including DNA electroporation, mRNA microinjection and whole-mount fluorescence in situ hybridization (FISH) of embryos (Christiaen et al., 2009a,b,c). The phylogenetic position of tunicates as our closest invertebrate relatives has also renewed interest in Ciona as a model organism (Delsuc et al., 2006). Ciona is a basal invertebrate chordate (phylum: Chordata, subphylum: Tunicata, class: Ascidiacea). Like all invertebrates, tunicates lack adaptive immune systems, and thus present the opportunity to study the innate immune system in isolation.

The Ciona tunic is a jelly-like coating that surrounds the animal mantle epithelium (Figure 1; De Leo et al., 1981, 1997). The thin, outermost layer of the tunic is a tough, fibrous cuticle composed mainly of cellulose. The interior of the tunic, often referred to as the tunic matrix or 'ground substance', is a thick, amorphous layer of cellulose fibrils and polysaccharides. Small tunicate cells of a variety of types are scattered throughout the tunic matrix. The Ciona tunic functions as an intermediary zone between the interior and exterior of the animal, 


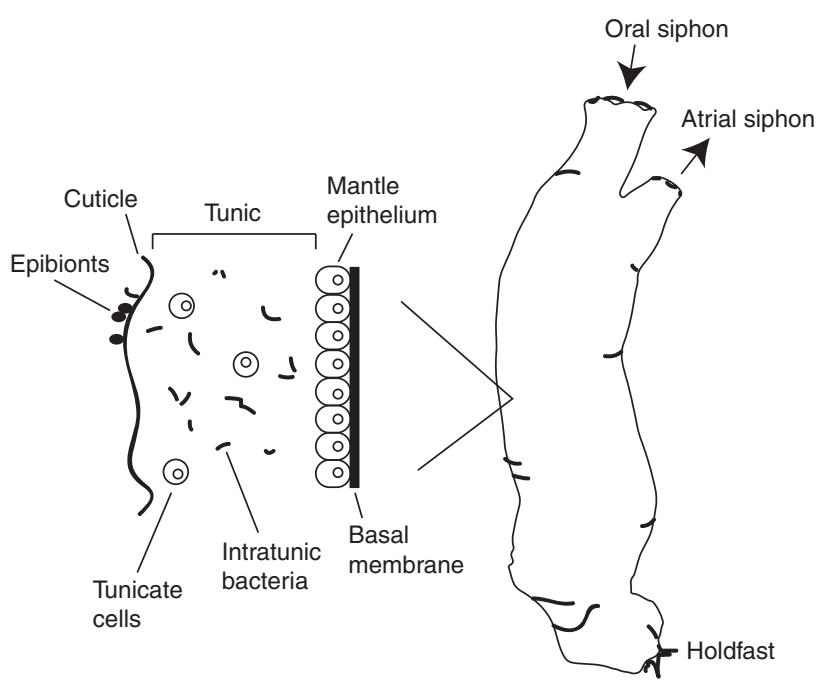

Ciona intestinalis

Figure 1 Schematic of $C$. intestinalis adult and a detail showing a cross-section of the tunic (redrawn after De Leo et al., 1997). The width of the tunic from mantle epithelium to cuticle is $\sim 200-400 \mu \mathrm{m}$ thick (De Leo et al., 1981).

similar to mucus layers in humans. As such, it serves as a site of self/non-self recognition and a barrier to infection (Di Bella and De Leo, 2000), making it attractive as a model for the role of innate immunity in establishment and maintenance of a surface-associated microbiome.

Tunicate bacterial communities are also of interest for their potential as sources of new natural products. The competitive and complex environment of sessile marine invertebrates may select for bacteria that can produce bioactive compounds. On average, 35 new natural products have been discovered from tunicates in each year since 2007 (Blunt et al., 2011), and many of these compounds are likely produced by microbial symbionts rather than the animal host (Schmidt and Donia, 2010). The uncultivated cyanobacterium Prochloron didemni has been recently shown to produce a suite of bioactive peptides including the patellamides and cyanobactins, which were originally isolated from the host tunicate Lissoclinum patella (Schmidt et al., 2005). In another example, the intracellular symbiont Candidatus Endoecteinascidia frumentensis has been implicated in production of the alkaloid ecteinascidin-743 from the tunicate Ecteinascidia turbinata (Rath et al., 2011). Even the model tunicate Ciona has yielded bioactive bacteria. The bacterium Pseudoalteromonas tunicata D2 was originally isolated from the surface of $C$. intestinalis (Holmstrom et al., 1998) and has been shown to produce a variety of bioactive molecules including the antifungal compound tambjamine, the purple pigment violacein and 3-formyl-tyrosine metabolites (Franks et al., 2005, 2006; Blasiak and Clardy, 2010).

This study aimed to characterize the tunic microbiome of adult $C$. intestinalis from Woods
Hole, MA, USA, to determine if there is a specific, tunic-associated community, and to identify the abundant phylotypes in and on the tunic. In order to provide a comprehensive analysis of the tunic bacterial community, we used a variety of techniques including 454 16S ribosomal RNA (rRNA) gene amplicon sequencing, microscopy and bacterial culture. We report the presence of several shared and dominant phylotypes across three $C$. intestinalis individuals. In addition, we succeeded in isolating four novel strains of tunic bacteria, all of which were abundant in the amplicon sequencing analysis. This is in contrast to many other environments in which cultured bacterial community members are rare within the total sequenced community (Shade et al., 2012). The combination of $16 \mathrm{~S}$ rRNA gene amplicon-based bacterial community analysis and available cultured isolates will provide a basis for future investigation of the ecological function of the tunic bacterial community.

\section{Materials and Methods}

C. intestinalis collection, dissection and DNA extraction

Initial microscopic observations of $C$. intestinalis tunic were performed on three adult individuals obtained from Woods Hole Marine Biological Laboratory (MBL) Marine Resource Center (MRC) aquaculture facility in July 2011. All further microscopy, 454 sequencing and bacterial isolation were performed on 15 wild-caught $C$. intestinalis adults directly collected from Eel Pond (41.526, - 70.670) by the MBL MRC in Woods Hole in December 2011. The water temperature at the collection site was $7^{\circ} \mathrm{C}$. Three $50 \mathrm{ml}$ Falcon tubes of seawater were collected at the site of the $C$. intestinalis collection. Specimens were shipped overnight to the Institute of Marine and Environmental Technology (IMET) in a large bag of seawater on ice and processed immediately on arrival. The seawater samples were syringe filtered with Sterivex-GV $0.22 \mu \mathrm{m}$ filter units (Millipore, Billerica, MA, USA). The filter units were opened and the filters were removed with sterile scalpels and stored at $-20^{\circ} \mathrm{C}$ until DNA extraction. Three additional $50 \mathrm{ml}$ water samples were taken from the collection bag containing the shipment of Ciona and were processed as described.

Multiple $\sim 0.5 \mathrm{~cm}^{3}$ tunic samples for DNA extraction and fixation were sterilely dissected from three C. intestinalis individuals. For tunic and cuticle samples (1-3TC), each section was rinsed $3 \times$ in sterile artificial seawater (ASW) and then processed for DNA extraction or fixation. For tunic interior samples (1-3T), the exterior cuticle was removed from the section with tweezers before rinsing $3 \times$ in sterile ASW. DNA was extracted from tunic samples or water filters using the Mo Bio PowerSoil DNA Isolation kit (Mo Bio, Carlsbad, CA, USA). The tunicate COI gene was sequenced from two 
individuals from Woods Hole, and these tunicates were confirmed to belong to $C$. intestinalis cryptic sp. B (Caputi et al., 2007; Supplementary Figure S1).

\section{Tunic fixation and catalyzed reporter deposition} (CARD)-FISH

Tunic samples were fixed overnight at $4{ }^{\circ} \mathrm{C}$ in $4 \%$ formaldehyde solution prepared from 16\% methanol-free formaldehyde (Thermo Scientific, Rockford, IL, USA) in ASW. Fixed samples were washed in ASW and incubated in $15 \%$ and then $30 \%$ sucrose in ASW for $1 \mathrm{~h}$ each at room temperature. Tunic samples were embedded in Tissue Tek OCT compound (Sakura, Torrence, CA, USA), flash-frozen in liquid $\mathrm{N}_{2}$ and sectioned to $8 \mu \mathrm{m}$ on a Tissue Tek $\mathrm{Cryo}_{3}$ microtome (Sakura). Sections were transferred to poly-L-lysine-coated slides (Electron Microscopy Sciences, Hatfield, PA, USA). CARD-FISH was performed according to established protocols (Pernthaler et al., 2002). Tunic sections were permeabilized for $30 \mathrm{~min}$ with $15 \mathrm{mg} \mathrm{ml}^{-1}$ lysozyme in $50 \mathrm{~mm}$ EDTA, $100 \mathrm{~mm}$ Tris pH 8 at $37^{\circ} \mathrm{C}$. Endogenous peroxidases were inactivated with $0.15 \% \mathrm{H}_{2} \mathrm{O}_{2}$ in methanol for $30 \mathrm{~min}$. Horseradish peroxidase-labeled probes were obtained from Biomers (Ulm, Germany) and used at a working concentration of $50 \mathrm{ng}^{-1} \mathrm{l}^{-1}$. Hybridizations were performed under established stringent hybridization conditions: $46{ }^{\circ} \mathrm{C}, 3 \mathrm{~h}$ hybridization, $35 \%$ formamide (EubI-III, Non338, CF319a) or $0 \%$ formamide (Euk516, Non338; Amann et al., 1990; Manz et al., 1996; Ishii et al., 2004). Amplification was performed using the Tyramide Signal Amplification system (Perkin Elmer, Waltham, MA, USA) with tyramides diluted 1:10 in provided amplification buffer. DNA was counterstained with 4',6-diamidino-2-phenylindole (DAPI). The hybridization stringency of the CF319a probe was confirmed by testing against alphaproteobacterial isolate CWH001 (negative control) and flavobacterium Tenacibaculum mesophilum MBIC 4357 (positive control). Bacterial cell density was calculated by direct counts of DAPI-stained objects in 10 fields of view in $8 \mu \mathrm{m}$ tunic sections. The percentage of CF319a or EubI-III-labeled cells relative to total DAPI-stained cells was calculated by comparing DAPI and Cy3 counts from 10 fields containing 35-115 cells.

\section{Culture and phylogenetic analysis of tunic-associated} bacteria

A $\sim 1 \mathrm{~cm}^{3}$ section of tunic from $C$. intestinalis individual 2 was pressed through a sterile $100 \mu \mathrm{m}$ nylon Cell Strainer (BD Falcon, Bedford, MA, USA) and diluted in $1 \mathrm{ml}$ sterile ASW. This solution was serially diluted in ASW and $100 \mu \mathrm{l}$ of the $10^{3}$ and $10^{5}$ dilutions were plated in triplicate on Difco Marine Agar 2216 (MA2216) (BD, Sparks, MD, USA) and natural seawater agar (autoclaved natural seawater with $1.5 \%$ agar) and observed for 2 weeks.
Four main morphotypes (CWH001, CWH003, CWH007 and CWH016) were subcultured to isolation on MA2216 at $20^{\circ} \mathrm{C}$. For DNA extraction and cryopreservation, tunic isolates were grown in $5 \mathrm{ml}$ Difco Marine broth 2216. Isolates were cryopreserved in $30 \%$ glycerol at $-80{ }^{\circ} \mathrm{C}$, but only CWH001 and CWH003 could be revived from the cryopreserved stock. DNA was extracted using the UltraClean Microbial DNA isolation kit (Mo Bio). The 16S rRNA gene was amplified and sequenced using primers 27F $5^{\prime}$-AGAGTTTGATCMTGGCTCAG - $3^{\prime}$ and 1492R 5'-TACGGYTACCTTGTTACGACTT-3' (Lane, 1991). The $50 \mu \mathrm{l}$ PCR reactions contained $\sim 50$ ng genomic DNA, 0.5 units Platinum Taq DNA polymerase (Invitrogen, Carlsbad, CA, USA) in supplied PCR Rxn buffer, $2 \mathrm{~mm} \mathrm{MgCl}_{2}$, $0.2 \mathrm{~mm}$ dNTP mix (Fermentas, Glen Burnie, MD, USA) and $0.2 \mu \mathrm{M}$ of each primer. The amplification conditions were as follows: $96{ }^{\circ} \mathrm{C}$ for $5 \mathrm{~min}$, followed by 25 cycles of $92{ }^{\circ} \mathrm{C}$ for $30 \mathrm{~s}, 46^{\circ} \mathrm{C}$ for $2 \mathrm{~min}$, and $72{ }^{\circ} \mathrm{C}$ for $1 \mathrm{~min}$ $30 \mathrm{~s}$, with a final extension at $72{ }^{\circ} \mathrm{C}$ for $10 \mathrm{~min}$. PCR products were sequenced on an ABI 3130 XL Genetic Analyzer (Applied Biosystems, Foster City, CA, USA) in IMET's BioAnalytical Services Laboratory.

16S rRNA gene sequences of isolates and their closest cultured and uncultured BLAST hits were aligned using the SILVA Incremental Aligner (SINA) v1.2.11 (Pruesse et al., 2012). Aligned sequences were added to 'The All-Species Living Tree' Project (LTP) database LTPs108 by maximum parsimony in ARB (Ludwig et al., 2004) and the alignment was further refined manually. Type strains representing closely related and representative groups were selected for phylogenetic analysis. A 50\% positional base frequency filter was calculated over either all Alphaproteobacteria or Bacteroidetes. The final alignments comprised $1262 \mathrm{bp}$ and $1211 \mathrm{bp}$ for the Alphaproteobacteria and Flavobacteria trees, respectively. Alignments were exported and analyzed with Phylip 3.69 (Felsenstein, 2005) to generate neighbor-joining and maximum parsimony consensus trees with $1000 \times$ bootstraps. Maximumlikelihood trees with $1000 \times$ bootstraps were calculated with PhyML 3.0 Online (Guindon et al., 2010) using the HKY85 substitution model with empirical base frequencies and estimated transition/ transversion ratio, invariable sites and gamma distribution parameter. There was good agreement between the neighbor-joining, maximum parsimony and maximum-likelihood trees with well-supported nodes consistent across all three methods.

\section{16S rRNA amplicon sequencing}

A total of six DNA samples from three Ciona individuals were analyzed by 454 sequencing of 16S rRNA amplicons. One DNA sample from tunic interior (T) and one from tunic and cuticle (TC) were sequenced from each individual. One DNA sample for each water type including water from the 
collection site (CW) and shipment bag (BW) were also analyzed. DNA concentrations ranged from 5 to $9 \mathrm{ng} \mathrm{ll}^{-1}$ as measured by Nanodrop 2000 spectrophotometer (Thermo Scientific). PCR amplification and 454 barcoded amplicon sequencing were performed by Research and Testing Laboratory (Lubbock, TX, USA) on the 454 Life Sciences GS FLX+ platform (Roche Diagnostics, Branford, CT, USA). The V1-V3 region of the 16S rRNA gene was amplified using primers 28F (barcoded 5'-GAGTTT GATCNTGGCTCAG-3') and 519R (5'-GTNTTACN GCGGCKGCTG-3'). Sample 1TC was amplified and sequenced twice as the first sequencing run generated $<2000$ reads. Only the second sequencing run of 1TC was included in the analysis. A complete list of samples and barcoded primers is available in Supplementary Table S1.

Data processing was performed in Mothur v1.28.0 (Schloss et al., 2009) and generally followed the procedures recommended in Schloss et al. (2011). Raw sequencing reads were denoised using the Pyronoise algorithm (Quince et al., 2011) and sequences were required to have exact barcode and primer matches, no ambiguous base calls, and no homopolymers $>8 \mathrm{bp}$. Barcode and primer sequences were removed and then sequences with $<250$ bp were discarded. Finally, sequences were all trimmed to exactly $250 \mathrm{bp}$ before analysis. Sequences were preclustered using an abundance weighted single-linkage preclustering step at $\sim 2 \%$ difference (maximum 2-bp difference over $250 \mathrm{bp}$ ) as originally recommended by Huse et al. (2010). Chimeras were identified and removed using the UChime algorithm (Edgar et al., 2011). Sequences were classified against the Ribosomal Database Project (RDP) database release 9 (Cole et al., 2009) with a bootstrap cutoff of $50 \%$ (Wang et al., 2007). Sequences that classified as chloroplasts were removed. A distance matrix was generated using pairwise.seqs in MOTHUR and clustering was performed using the average neighbor method at $3 \%$ to generate operational taxonomic units (OTUs). Details of read numbers following each processing step are given in Supplementary Table S2.

Near complete 16S rRNA gene sequence for OTU2 was obtained using the specific primer A1CWF $5^{\prime}-\mathrm{G}$ GAATCTACCTTATAGTGGGGGATAACTTC- $3^{\prime}$ along with $1492 \mathrm{R}$ for amplification from sample $2 \mathrm{~T}$ as described for $27 \mathrm{~F} / 1492 \mathrm{R}$ but with annealing at $55^{\circ} \mathrm{C}$. Sequencing was performed using specific primers A1CWF and A1CWF_mid 5'-GCTAAACGTCGGGTC TTAGGAT-3'.

Sequence data are available in the GenBank database under accession no. KF494349-55 and the MG-RAST database under project 5387 with metagenome IDs 4530382.3-89.3.

\section{Results}

Tunic microbiome analysis

DNA from six water samples and three tunicate individuals was used to create $16 \mathrm{~S}$ rRNA gene amplicon libraries that were initially screened by denaturing gradient gel electrophoresis (DGGE) to compare the bacterial communities (Supplementary Methods and Supplementary Figure S2). Based on this analysis, two water samples and six tunicate samples were selected for 454 sequencing. Two samples were sequenced from each tunicate individual, one sample of tunic and exterior cuticle (TC), and one sample containing only interior tunic (T). DNA from associated seawater samples was sequenced from both the collection site (CW) and the shipping bag (BW). The 454 amplicon libraries were randomly subsampled to 2500 sequences per sample, which provides nearly complete coverage (97-99\% Good's coverage) of tunic libraries at 3\% OTU clustering (Supplementary Table S3). The 16S rRNA gene libraries from water samples were more diverse than those of the tunic samples as assayed by both DGGE and 454 sequencing, with an average of 51 OTUs detected in the tunic samples and 526 OTUs and 278 OTUs detected in the collection bag water and seawater respectively (Figure 2, Supplementary Figure S3 and Supplementary Table S3). The number of OTUs detected in tunic samples ranged from 15 to 147 . In addition, the tunic and cuticle samples had greater richness than the tunic-only samples from the same individual, with 15-22 OTUs observed for tunic-only samples and 29-147 OTUs observed for tunic and cuticle samples (Supplementary Table S3 and Supplementary Figure S3).

The sequences in the tunic 16S rRNA gene fragment libraries were classified by RDP into three main phyla, Proteobacteria, Bacteroidetes and unclassified Bacteria (Figure 2a). The tunic Proteobacteria sequences belonged mainly to Alphaproteobacteria or could not be further classified (unclassified_Proteobacteria), but all other classes within the Proteobacteria were detected in at least one tunic sample. Actinobacteria, Planctomycetes, Firmicutes, Verrucomicrobia, Chloroflexi and Armatimonadetes were all also present in low abundance in at least one tunicate. Only tunicate individual three (both 3T and 3TC) contained 1-3\% of sequences from an OTU categorized as 'unclassified bacteria' by RDP. Alignment of a representative sequence from this OTU to the SILVA SSU reference database indicated that this OTU groups with the candidate division BD15. Sequences from the phylum Cyanobacteria comprised $2 \%$ of collection seawater sequences (CW) and $33 \%$ of shipping bag seawater sequences (BW) but were not detected in any tunic DNA library. In addition, the bacterial groups TM7, Acidobacteria, Fusobacteria, Spirochaetes, Nitrospira and OD1 were detected in seawater amplicon libraries but not in tunic libraries.

Only nine OTUs contained $>1 \%$ of the total tunicate sequences and accounted for $>90 \%$ of the total tunicate sequences (Figure 3). These OTUs were classified as Flavobacteria, unclassified 
a

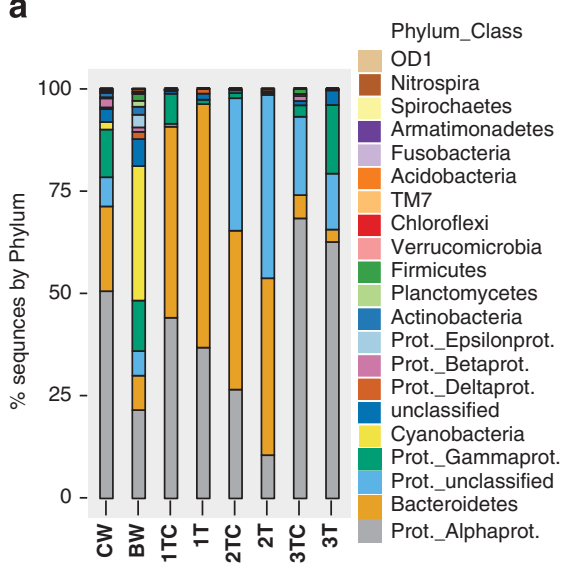

b

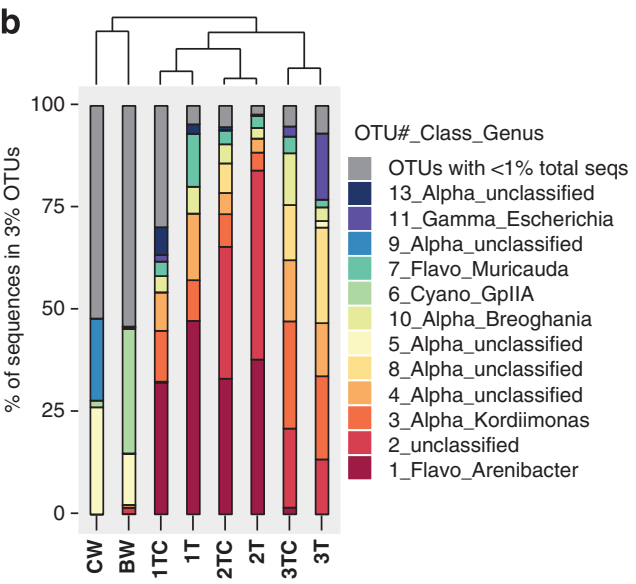

Figure 2 Bacterial community analysis by 454 sequencing of $16 \mathrm{~S}$ rRNA genes. Sample names are: collection site seawater (CW), shipping bag seawater (BW), C. intestinalis individuals 1-3 tunic and cuticle (1-3TC) or tunic only (1-3T). (a) Relative abundance of sequences classified to the phylum level by RDP. Proteobacteria are expanded to the class level. (b) Relative abundance of sequences grouped into 3\% OTUs. A representative sequence from each OTU was used for RDP classification. Rare taxa (OTUs containing < $1 \%$ of total sequences) are grouped together. Dendrogram at top of figure shows UPGMA clustering of the samples based on the Yue and Clayton estimator of similarity, $\theta$, which compares community structures.

Proteobacteria, Alphaproteobacteria and Gammaproteobacteria (Table 1). Using specific primers that target the OTU 2 sequence, the second-most abundant group in tunicate samples, we were able to obtain a near complete 16S rRNA gene sequence for this group, and RDP classification indicates that it also belongs to the Alphaproteobacteria. The eight most abundant OTUs were detected in all tunicate individuals. Hence, the most abundant OTUs were also the most commonly shared OTUs. Less common tunicate OTUs were only detected in one or two tunicates.

Beta diversity analysis demonstrated that the bacterial community structures of the water and tunicate samples were clearly different (Figure 2b). The water samples consistently grouped separately from the tunicate samples in UPGMA clustering based on the Yue and Clayton $\theta$ estimator and Bray Curtis similarity metrics (Schloss et al., 2009). A clustering analysis of DGGE banding patterns also supported separate clustering of the water and tunicate bacterial communities (Supplementary Figure S2). Within the tunicate samples, the bacterial communities clustered by tunicate individual rather than by sample type (interior tunic only vs tunic and cuticle). The bacterial community of tunicate 3 was most different, with fewer reads corresponding to OTU 1 (Flavobacteria_Arenibacter) and more corresponding to proteobacterial OTUs (Figure 3). The clustering of DGGE banding patterns of samples from tunicate 3 provided a similar outcome (Supplementary Figure S2).

Bacterial culture from C. intestinalis tunic

Four main bacterial morphotypes were isolated from C. intestinalis tunic and $16 \mathrm{~S}$ rRNA gene sequencing demonstrated that these four morphotypes each corresponded to an abundant OTU in the 454 data set (Table 1). Three of the isolates also had $100 \%$ identity to sequenced bands from the DGGE analysis (Supplementary Figure S2). ML trees showing the position of the four new bacterial isolates in relation to their closest BLAST hits and reference strains are shown in Figure 4. Isolate CWH001, corresponding to the third-most abundant tunicate OTU, clusters within the alphaproteobacterial Kordiimonas genus and shares $95 \%$ identity with type strain Kordiimonas gwangyangensis. RDP classification of isolate CWHO03 (OTU 8) supports affiliation within the alphaproteobacterial genus Kiloniella (confidence $84 \%$ ); however, the SINA classifier assigns this sequence to the Rhodospiralles order and the Thalassospira genus. CWH003 shares only 90\% identity with the type strain Kiloniella laminariae. The phylogenetic analysis does not give strong support for affiliation with any characterized genera, but CWH003 does group consistently with the Kiloniella and Pelagibius genera rather than within the Thalassospira. The isolate CWH007 has $95 \% 16 \mathrm{~S}$ rRNA sequence identity to type strain Flagellimonas eckloniae and $96 \%$ identity to type strain Muricauda olearia CL-SS4. The RDP classifier assigns CWH007 (OTU 7) to the Muricauda genus with $100 \%$ confidence. The phylogenetic analysis shows CWH007 clustering consistently within a group of Flavobacteriaceae including the Muricauda, Croceitalea and Flagellimonas genera, but does not support clear affiliation with any genus. Isolate CWH016, corresponding to the most abundant tunicate OTU 1, groups with strong support within the flavobacterial genus Arenibacter, and this isolate has $94 \% 16 \mathrm{~S}$ rRNA gene sequence identity to the type strain Arenibacter palladensis. 


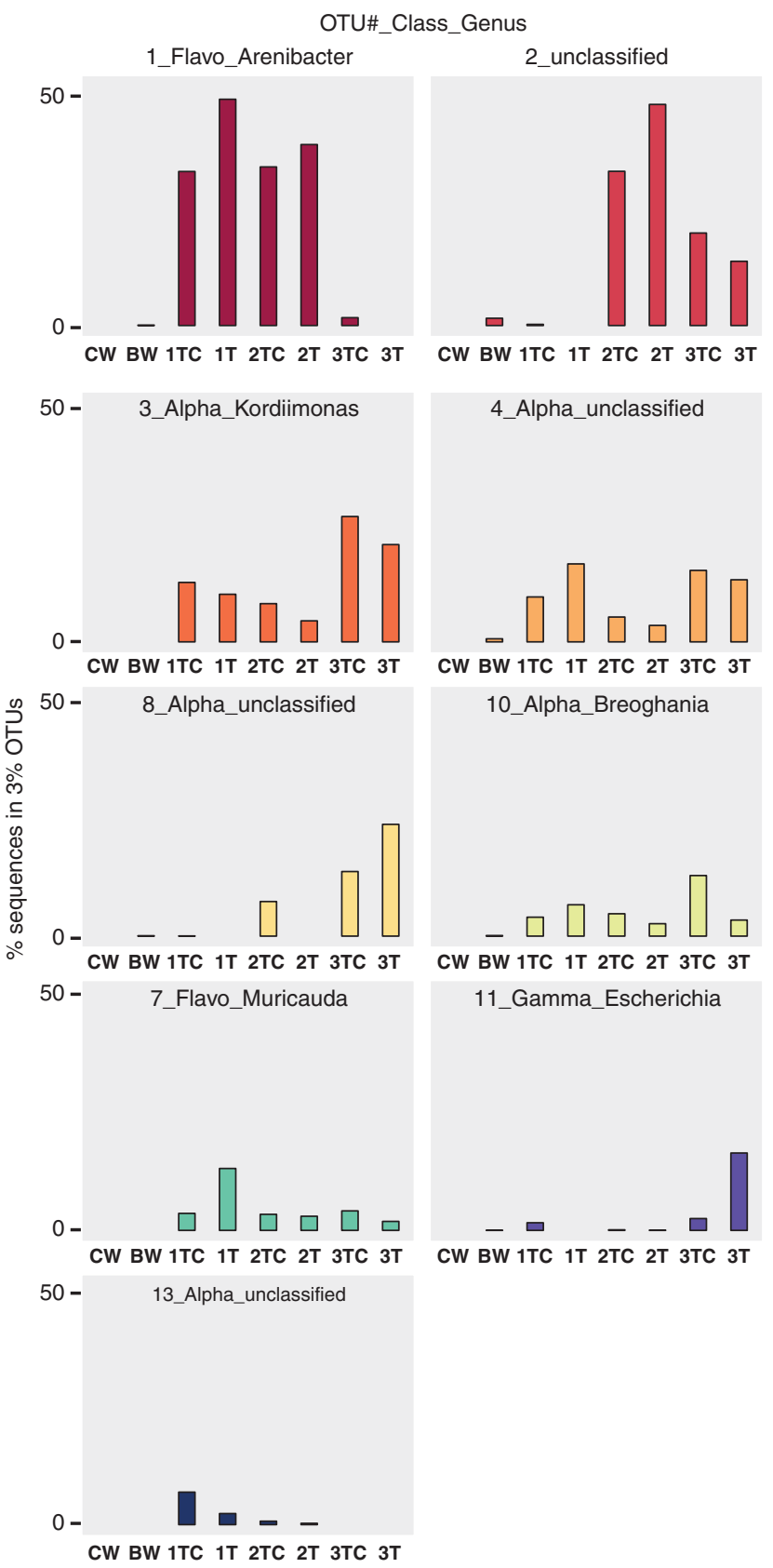

Figure 3 Expanded view of Figure $2 b$ to allow direct comparison across samples. Each panel shows the relative abundance of sequences within an OTU. Only OTUs containing $>1 \%$ of the total reads from tunicate samples are shown.

CARD-FISH of bacteria in C. intestinalis tunic Microscopy of Gram-stained tunic sections revealed an even, dense population of Gram-negative bacteria embedded throughout the tunic matrix, interspersed with a variety of tunicate cells (Figure 5a). General bacterial and eukaryotic CARD-FISH probes were used to visualize bacteria and tunicate cells within tunic sections (Figures $5 \mathrm{~b}$ and $\mathrm{c}$ ). The interior tunic contains $1-2 \mu \mathrm{m}$ bacteria evenly spaced within the tunic matrix, with few, spherical $\sim 10 \mu \mathrm{m}$ diameter tunicate cells. The majority of the interior tunic bacterial population was labeled by the specific CF319a Cytophaga-Flavobacteria probe, which is an exact match to both isolates CWH016 (OTU 1) and CWH007 (OTU 7) (Figure 5d and e). By directly counting DAPI-stained bacterial cells within tunic sections, we estimated $2.6 \pm 0.9 \times 10^{9}$ cells cm $^{-3}$ for individual 1, $2.8 \pm 0.6 \times 10^{9} \mathrm{cells} \mathrm{cm}^{-3}$ for individual 2 and $8.8 \pm 0.2 \times 10^{9}$ cells $\mathrm{cm}^{-3}$ for individual 3 (sample s.d. are indicated). The percentage of cells specifically labeled by CARD-FISH was quantified for individual 2 by comparing Cy3-labeled cells with total DAPI counts. The EubI-III probe detected an average of $78 \pm 10 \%$ of the DAPI-stained cells, whereas the specific Cytophaga-Flavobacteria probe CF319a labeled $37 \pm 9 \%$ of cells within the tunic.

\section{Discussion}

The presence of bacteria within the tunic of C. intestinalis was first reported over 30 years ago with electron microscopy images showing bacilli of $\sim 2 \mu \mathrm{m}$ within tunic sections (De Leo et al., 1981). In a separate study, De Leo and Patricolo (1980) also observed a specific association with a probable cyanobacterial symbiont in $C$. intestinalis from tropical waters. Since these initial reports, only a few studies have remarked on the tunic bacteria (De Leo et al., 1997; Di Bella et al., 2011), and their phylogeny had never been investigated. Here we described the first culture and non-culture-based investigation of the Ciona tunic bacterial community.

Analysis of the beta diversity of tunic bacterial communities based on 454 sequencing of 16S rRNA amplicons showed that variation between individuals were greater than the variation between the tunic-only samples ( $\mathrm{T}$ ) and the tunic and exterior cuticle samples (TC). That is, the samples clustered by individual rather than by whether or not they contained exterior cuticle. For example, the bacterial community of 1T was more similar to that of 1TC, than to $2 \mathrm{~T}$ or $3 \mathrm{~T}$. This was surprising, given that we anticipated that different bacterial groups might be important on the interior and exterior of the tunic. We expected that surface-associated epibionts would be found only in the TC samples, while bacteria living within the interior tunic matrix would be found in both $\mathrm{T}$ and $\mathrm{TC}$ samples. These individual bacterial community differences observed in the sequence data were also supported by $16 \mathrm{~S}$ rRNA DGGE analysis using a different primer set. One possible explanation for these differences is simply that the dissection and removal of the exterior bacteria from $\mathrm{T}$ samples was imperfect, minimizing apparent community differences between the interior and exterior of the tunic. This explanation is unlikely to completely explain the observed differences as the TC samples had consistently higher measures of richness than their $\mathrm{T}$ 
Table 1 Classification of abundant tunic OTUs

\begin{tabular}{|c|c|c|c|c|c|c|}
\hline \multirow[t]{2}{*}{ OTU\# } & \multirow[t]{2}{*}{ Isolate } & \multirow{2}{*}{$\begin{array}{l}\text { RDP classification } \\
\text { class/genus }\end{array}$} & \multicolumn{4}{|c|}{ Closest uncultured and cultured BLAST hits } \\
\hline & & & Acc. \# & & Source & $\begin{array}{l}\operatorname{Max} . I d \\
(\%)\end{array}$ \\
\hline $1^{*}$ & CWH016 & Flavobacteria/Arenibacter & DQ486485 & Arenibacter sp. DG1238 & Dinoflagellate & 95 \\
\hline \multirow[t]{2}{*}{$2^{*}$} & - & Alphaprot./unclassified1 & HQ326311 & Uncultured clone SBS-RV-031 & Biofilm & 99 \\
\hline & & & DQ167245 & $\begin{array}{l}\text { Kopriimonas byunsanensis } \\
\text { KOPRI } 13522\end{array}$ & Marine biofilm & 88 \\
\hline \multirow[t]{2}{*}{$3^{*}$} & CWH001 & Alphaprot./Kordiimonas & EU287361 & Uncultured bacterium clone S26-61 & Arctic sediment & 99 \\
\hline & & & GU289640 & Kordiimonas sp. MEBiC06554 & Seawater & 96 \\
\hline \multirow[t]{2}{*}{4} & - & Alphaprot./unclassified2 & HQ163356 & Uncultured Alphaprot. SHZW738 & Seawater & 92 \\
\hline & & & JQ085424 & alphaproteobacterium NH6-42 & Seawater & 85 \\
\hline \multirow[t]{2}{*}{$8^{*}$} & CWH003 & Alphaprot./Kiloniella & EU2287341 & Uncultured bacterium clone S26-41 & Arctic sediment & 99 \\
\hline & & & AB571945 & Alphaproteobacterium AKA07-5 & - & 96 \\
\hline \multirow[t]{2}{*}{10} & - & Alphaprot./Breoghania & FJ543036 & Uncultured Alphaprot. B07-10D & Earthworm gut & 88 \\
\hline & & & GQ272328 & Alphaproteobacterium UBF-P1 & Sand after oil spill & 86 \\
\hline \multirow[t]{2}{*}{$7^{*}$} & CWH007 & Flavobacteria/Muricauda & FJ203619 & Uncultured bacterium clone SHFH709 & Coral & 98 \\
\hline & & & FJ348472 & Flavobacteriaceae bacterium A9 & Marine sponge & 97 \\
\hline \multirow[t]{2}{*}{11} & - & Gammaprot./Escherichia & КС002412 & Marine bacterioplankton clone & Seawater & 100 \\
\hline & & & JX120100 & P4-3B_16 Bacterium P618/ & Swine feces & 100 \\
\hline \multirow[t]{2}{*}{13} & - & Alphaprot./unclassified3 & JF297198 & Uncultured Hyphomicrobiaceae & Lobster shell legion & 98 \\
\hline & & & EF554909 & Devosia sp. G-He10 & - & 87 \\
\hline
\end{tabular}

Abbreviations: OTU, operational taxonomic unit; RDP, Ribosomal Database Project; rRNA, ribosomal RNA.

Top BLAST hits and closest cultured BLAST hits for the nine OTUs with $>1 \%$ of total 45416 rRNA gene sequence abundance from tunicate samples. When available, almost complete $16 \mathrm{~S}$ sequence was used for RDP classification and BLAST searches as indicated by an asterisk. BLAST search was performed on 18 January 2013.

counterparts, indicating that some groups occur only in the exterior samples. Alternatively, most bacterial groups that occur on the tunic exterior may also be present within the tunic interior matrix. Finally, similarity of community structure within individual tunic samples may reflect a true variation of the tunic microbiome among individuals. This is similar to what has been observed in the human microbiome, where each individual has a unique microbiome, and as sequencing effort of individuals increases, the core of shared taxa decreases, although functional traits of the community may be conserved (Turnbaugh et al., 2009; Consortium, 2012).

Although the bacterial communities of each Ciona individual were different, the most abundant amplicons were consistently detected across all three tunicate individuals. The observed tunic bacterial communities contain dominant phylotypes that are shared between individuals and enriched compared with the surrounding seawater. The diversity of the observed $C$. intestinalis tunic microbiome was much lower than the diversity of the vertebrate-associated microbiome. For example, the microbiome of human gut and skin are expected to contain $\sim 30000$ and $\sim 2000$ OTUs at 3\% clustering (Huse et al., 2012) compared with the 15-150 OTUs observed in Ciona tunic samples. Low diversity has also been observed in other invertebrate microbiomes including those associated with the cnidarian Hydra (Fraune and Bosch, 2007) and the leech (Kikuchi and Graf, 2007).
The observation of shared and abundant phylotypes across these tunicate samples raises questions about whether these resident phylotypes are conserved across time and space. The three tunicate individuals sequenced here were collected from a single site in December 2011, and future study will be required to determine the stability of the tunic bacterial community with respect to different $C$. intestinalis populations (cryptic subsp. A vs B described here), geography, seasons, environmental conditions and health. In addition, although Ciona is a solitary tunicate, it is often found growing in large clusters of individuals, which could facilitate future investigation of the effect of proximity and genetic diversity on the associated microbiome.

The Ciona tunic has several advantages as a potential model system for extracellular or host surface-associated bacterial communities, including the relative simplicity of the resident community, with $<10$ abundant phylotypes observed in these individuals, and the ability to culture representatives of at least 4 of these abundant groups. Our initial culture effort was minimal, and we expect that more extensive culturing efforts with more media types and longer incubation times could yield more cultivable diversity. Future experimental work will be required to characterize the ecological role of these isolates within the tunic community and determine if they have functional importance. The isolate CWH001, which corresponds to OTU3, like all of the Kordiimonas spp. that have been described 

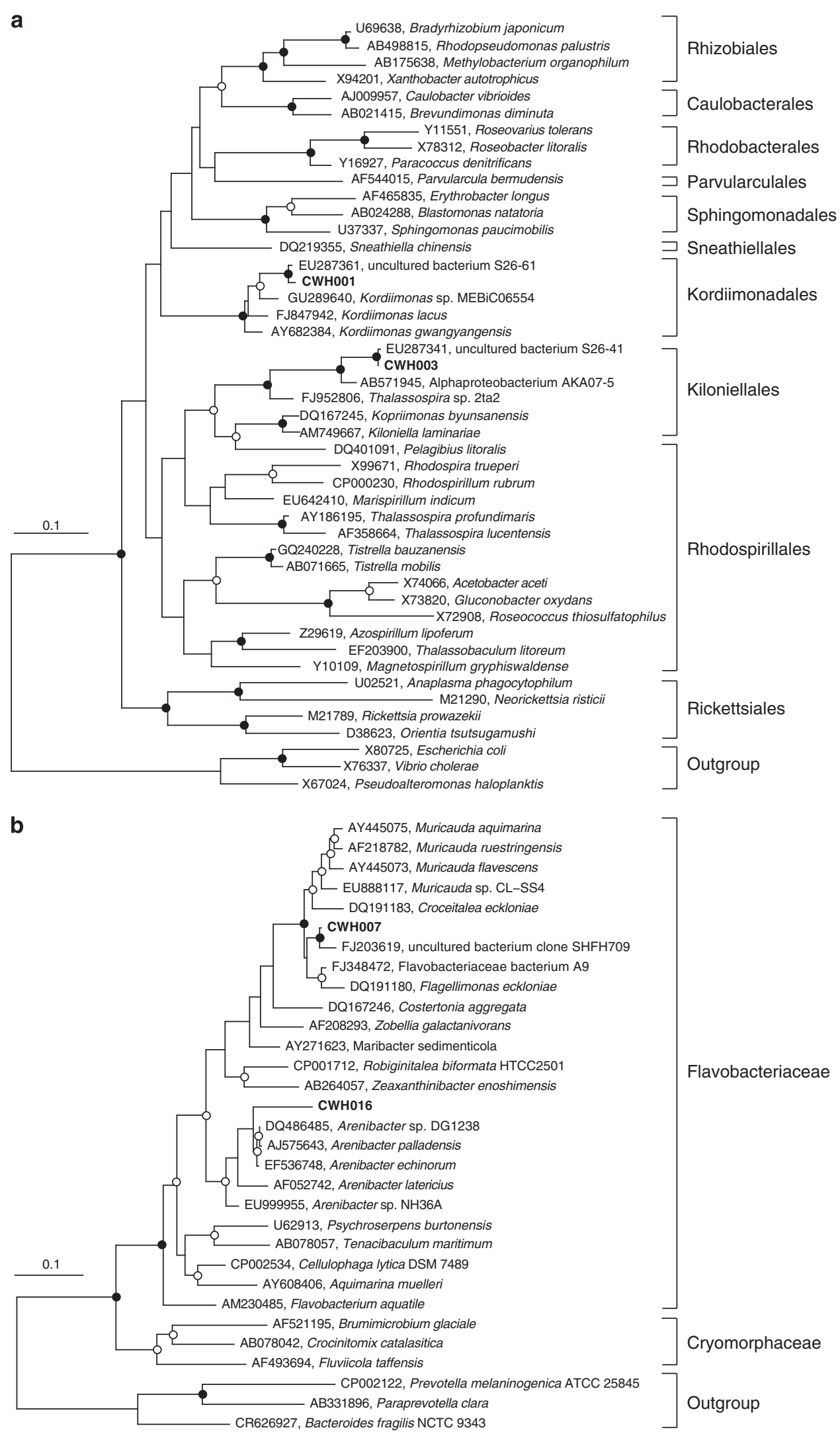

Figure 4 Maximum likelihood trees with $1000 \times$ bootstrap resampling of Alphaproteobacteria (a) and Flavobacteria (b) isolates from C. intestinalis tunic. Sequences from this study are shown in bold. Nodes with $>90 \%$ bootstrap support are indicated by closed circles and nodes with $>50 \%$ support by open circles. 

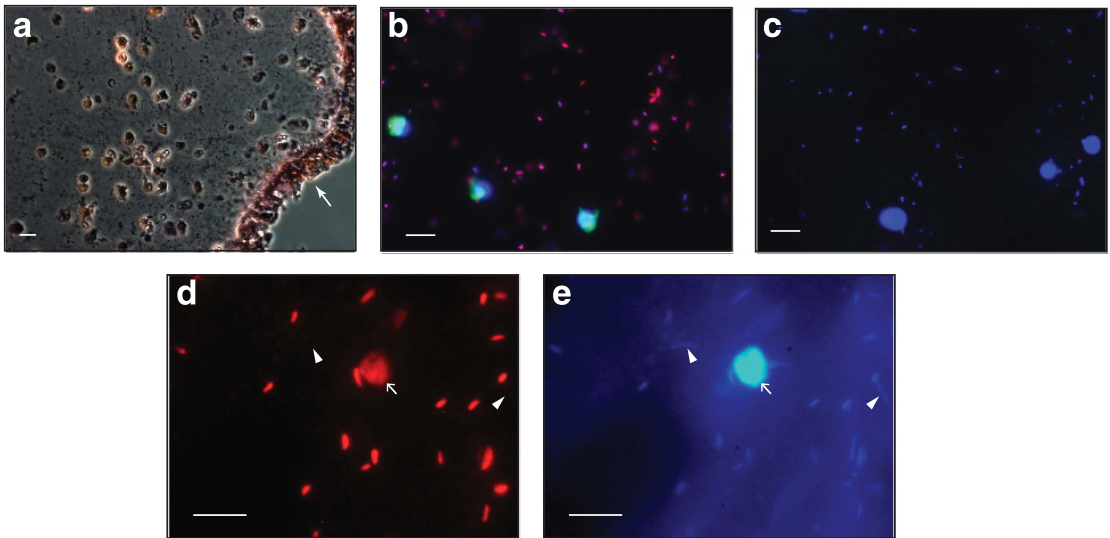

Figure 5 Epifluorescent micrographs of CARD-FISH-labeled bacteria within the C. intestinalis tunic. (a) Gram-stained tunic and cuticle section. White arrow denotes exterior cuticle. (b) C. intestinalis individual 5 from Woods Hole July 2011 collection. EubI-III-labeled bacteria (red), Euk516-labeled tunicate cells (green) and DAPI-stained DNA (blue). (c) Same tunicate individual as in a with double Non338 (red, green) negative control showing no hybridization. DNA is stained with DAPI (blue). (d) C. intestinalis individual 2 from Woods Hole December 2011 collection. CF319a-labeled Flavobacteria-Cytophaga bacteria (red). Unlabeled bacteria are indicated with white arrowheads. White arrow shows tunicate cell. (e) Same image as C showing DAPI-stained DNA (blue). White scale bars are $10 \mu \mathrm{m}$.

thus far, is an aerobic, marine bacterium. Members of the Kordiimonas genus have also been detected through culture-independent methods in the marine sponge Axinella corrugata (GenBank accession EF092170) as well as in the bacteria associated with the accessory nidamental gland of several cephalopod species (Pichon et al., 2005; Collins et al., 2012). These nidamental bacteria are thought to be vertically transmitted to the cephalod eggs or egg sac and to function in some protective capacity. Interestingly, cephalopod nidamental bacteria have also been shown to include flavobacterial groups (Collins et al., 2012), and clone libraries from the squid Loligo pealei egg capsule contained several sequences that were closely related to the alphaproteobacterial OTU 8/isolate CWH003 (Barbieri et al., 2001).

Despite mention of the presence of some intratunic bacteria in several $C$. intestinalis studies (De Leo et al., 1981, 1997; Di Bella et al., 2011), it was quite surprising to observe such a dense bacterial population within the $C$. intestinalis tunic. Bacteria were observed throughout the tunic in all specimens of the Woods Hole C. intestinalis population that we examined (multiple individuals across both summer and winter collections). Similarly dense intratunic bacteria are commonly observed in colonial tunicates, including the Synoicum adareanum and Lissoclinum patella (Riesenfeld et al., 2008; Donia et al., 2011). Although the $C$. intestinalis specimens used in this study appeared to be healthy adults and the bacteria were observed spread evenly throughout the tunic tissue, we cannot completely discount the possibility that the observed tunic bacteria represent a pathogenic infection. Alternatively, the tunic bacteria could function to protect the tunicate against fouling or pathogens through production of natural products, chemical signals or competition. In future work, investigation of the tunic microbiome across larger populations, geographic regions and tunicate life stages could begin to provide clues about the stability and ecological roles of these bacterial associations.

The tunic microbiome is of special interest given that Ciona is re-emerging model system for the study of innate immunity in a basal chordate. Invertebrate model systems have revealed a surprising specificity and complexity in these host-microbe associations, despite the lack of an adaptive immune system (reviewed in Nyholm and Graf, 2012). For example, study of the Vibrio fisheri-squid symbiosis demonstrated that recognition of microbial-associated molecular patterns can mediate symbiosis or immune response depending on the context (Koropatnick et al., 2004). The $C$. intestinalis genome encodes only two Toll-like receptor homologs, which both activate the transcription factor nuclear factor-KB in direct response to multiple microbial-associated molecular patterns (Sasaki et al., 2009). C. intestinalis has also been shown to express a complement gene, CiC3-1, in hemocytes (Pinto et al., 2003), and opsonins termed variable region-containing chitin binding proteins are expressed in the gut epithelium and hemocytes (Dishaw et al., 2011). Ciona mounts an inflammatory response when the tunic is injured through injection of seawater, Escherichia coli lipopolysaccharide, or sheep erythrocytes (Parrinello et al., 1977, 2007). This response includes a visible encapsulation reaction at the injured site and activation of the prophenoloxidase system and melanization (De Leo et al., 1997; Cammarata et al., 2008). Several antimicrobial peptides are also expressed in tunic granulocytes in as part of the 
tunic inflammatory response (Di Bella et al., 2011). The results presented in this study, along with our ability to culture major phylotypes, provide new insights into the Ciona tunic bacterial community and lay the foundation to study the interactions between the observed tunic bacteria and their hosts.

\section{Conclusions}

The tunic-associated bacterial community of C. intestinalis is distinct from that of the surrounding seawater and contains an abundant alphaproteobacterial and flavobacterial phylotypes. A dense population of tunic bacteria living within the tunic interior was observed by CARD-FISH across multiple individuals and collections, and was comprised of mainly flavobacteria. Although $C$. intestinalis is a well-studied model organism, a minimal culturing effort yielded several novel bacterial isolates that will be interesting to investigate for their natural product potential. Unlike in many other systems, these culturable bacteria were also abundant members of the tunic-associated community as assayed by $16 \mathrm{~S}$ rRNA gene-based methods. The availability of these dominant community members in culture will be a valuable resource for interrogating their role with respect to the tunicate host. The $C$. intestinalis tunic bacterial community represents an exciting model system in which to study the interplay between the host microbiome and innate immunity in development.

\section{Conflict of Interest}

The authors declare no conflict of interest.

\section{Acknowledgements}

Funding was provided by the National Science Foundation (NSF) Directorate for Biological Sciences Integrative Organismal Systems (IOS-0919728), Fogarty International Center, National Cancer Institute, National Institute of Allergy and Infectious Diseases, National Institute of Mental Health, National Institute on Drug Abuse, National Heart Lung and Blood Institute, National Center for Complementary and Alternative Medicine, Office of Dietary Supplements, National Institute of General Medical Sciences, Biological Sciences Directorate of the NSF, and the Office of Biological and Environmental Research of the US Department of Energy under Cooperative Agreement U01 TW00313 with the International Cooperative Biodiversity Groups. Leah C Blasiak was supported by the Selman A Waksman Endowed Scholarship in Microbial Diversity funded by the Gordon and Betty Moore foundation while a participant in the Marine Biological Laboratories Microbial Diversity Course in Woods Hole. We thank Sara Kleindienst, Charles Pepe-Ranney, and Elizabeth Wilbanks for valuable input, and the MBL for use of its LSCM facilities. This is IMET contribution no. 13-111 and UMCES contribution no. 4812.

\section{References}

Amann RI, Binder BJ, Olson RJ, Chisholm SW, Devereux R, Stahl DA. (1990). Combination of $16 S$ rRNAtargeted oligonucleotide probes with flow cytometry for analyzing mixed microbial populations. Appl Environ Microbiol 56: 1919-1925.

Barbieri E, Paster BJ, Hughes D, Zurek L, Moser DP, Teske A et al. (2001). Phylogenetic characterization of epibiotic bacteria in the accessory nidamental gland and egg capsules of the squid Loligo pealei (Cephalopoda:Loliginidae). Environ Microbiol 3: 151-167.

Blasiak LC, Clardy J. (2010). Discovery of 3-formyltyrosine metabolites from Pseudoalteromonas tunicata through heterologous expression. J Am Chem Soc 132: 926-927.

Blunt JW, Copp BR, Munro MH, Northcote PT, Prinsep MR. (2011). Marine natural products. Nat Prod Rep 28: 196-268.

Cammarata M, Arizza V, Cianciolo C, Parrinello D, Vazzana M, Vizzini A et al. (2008). The prophenoloxidase system is activated during the tunic inflammatory reaction of Ciona intestinalis. Cell Tissue Res 333: 481-492.

Caputi L, Andreakis N, Mastrototaro F, Cirino P, Vassillo M, Sordino P. (2007). Cryptic speciation in a model invertebrate chordate. Proc Natl Acad Sci USA 104: 9364-9369.

Christiaen L, Wagner E, Shi W, Levine M. (2009a). Wholemount in situ hybridization on sea squirt (Ciona intestinalis) embryos. Cold Spring Harb Protoc 2009; doi:10.1101/pdb.prot5348.

Christiaen L, Wagner E, Shi W, Levine M. (2009b). Electroporation of transgenic DNAs in the sea squirt. Ciona. Cold Spring Harb Protoc 2009; doi:10.1101/ pdb.prot5345.

Christiaen L, Wagner E, Shi W, Levine M. (2009c). Microinjection of morpholino oligos and RNAs in sea squirt (Ciona) embryos. Cold Spring Harb Protoc 2009; doi:10.1101/pdb.prot5347.

Cole JR, Wang Q, Cardenas E, Fish J, Chai B, Farris RJ et al. (2009). The ribosomal database project: improved alignments and new tools for rRNA analysis. Nucleic Acids Res 37: D141-D145.

Collins AJ, LaBarre BA, Won BS, Shah MV, Heng S, Choudhury MH et al. (2012). Diversity and partitioning of bacterial populations within the accessory nidamental gland of the squid Euprymna scolopes. Appl Environ Microbiol 78: 4200-4208.

Consortium H. (2012). Structure, function and diversity of the healthy human microbiome. Nature 486: 207-214.

De Leo G, Patricolo E. (1980). Blue-green algalike cells associated with the tunic of Ciona intestinalis L. Cell Tissue Res 212: 91-98.

De Leo G, Patricolo E, Frittitta G. (1981). Fine Structure of the Tunic of Ciona intestinalis. Acta Zoologica 62: 259-271.

De Leo G, Parrinello N, Parrinello D, Cassara G, Russo D, Di Bella MA. (1997). Encapsulation response of Ciona intestinalis (Ascidiacea) to intratunical erythrocyte injection. J Invertebr Pathol 69: 14-23.

Dehal P, Satou Y, Campbell RK, Chapman J, Degnan B, De Tomaso A et al. (2002). The draft genome of Ciona intestinalis: insights into chordate and vertebrate origins. Science 298: 2157-2167.

Delsuc F, Brinkmann H, Chourrout D, Philippe H. (2006). Tunicates and not cephalochordates are the closest living relatives of vertebrates. Nature 439: 965-968. 
Di Bella MA, De Leo G. (2000). Hemocyte migration during inflammatory-like reaction of Ciona intestinalis (Tunicata, ascidiacea). J Invertebr Pathol 76: 105-111.

Di Bella MA, Fedders H, Leippe M. (2011). Localization of antimicrobial peptides in the tunic of Ciona intestinalis (Ascidacea, Tunicata) and their involvement in local inflammatory-like reactions. Results Immunol 1: 70-75.

Dishaw LJ, Giacomelli S, Melillo D, Zucchetti I, Haire RN, Natale L et al. (2011). A role for variable regioncontaining chitin-binding proteins (VCBPs) in host gut-bacteria interactions. Proc Natl Acad Sci USA 108: 16747-16752.

Donia MS, Fricke WF, Partensky F, Cox J, Elshahawi SI, White JR et al. (2011). Complex microbiome underlying secondary and primary metabolism in the tunicate-Prochloron symbiosis. Proc Natl Acad Sci USA 108: E1423-E1432.

Edgar RC, Haas BJ, Clemente JC, Quince C, Knight R. (2011). UCHIME improves sensitivity and speed of chimera detection. Bioinformatics 27: 2194-2200.

Felsenstein J. (2005). PHYLIP (Phylogeny Inference Package) version 3.6. Distributed by the author Department of Genome Sciences, University of Washington: Seattle, WA, USA

Franks A, Egan S, Holmstrom C, James S, Lappin-Scott H, Kjelleberg S. (2006). Inhibition of fungal colonization by Pseudoalteromonas tunicata provides a competitive advantage during surface colonization. Appl Environ Microbiol 72: 6079-6087.

Franks A, Haywood P, Holmstrom C, Egan S, Kjelleberg S, Kumar N. (2005). Isolation and structure elucidation of a novel yellow pigment from the marine bacterium Pseudoalteromonas tunicata. Molecules 10: $1286-1291$.

Fraune S, Bosch TC. (2007). Long-term maintenance of species-specific bacterial microbiota in the basal metazoan. Hydra. Proc Natl Acad Sci USA 104: 13146-13151.

Guindon S, Dufayard JF, Lefort V, Anisimova M, Hordijk W, Gascuel O. (2010). New algorithms and methods to estimate maximum-likelihood phylogenies: assessing the performance of PhyML 3.0. Syst Biol 59: 307-321.

Holmstrom C, James S, Neilan BA, White DC, Kjelleberg S. (1998). Pseudoalteromonas tunicata sp. nov., a bacterium that produces antifouling agents. Int $J$ Syst Bacteriol 48 (Pt 4): 1205-1212.

Huse SM, Welch DM, Morrison HG, Sogin ML. (2010). Ironing out the wrinkles in the rare biosphere through improved OTU clustering. Environ Microbiol 12: 1889-1898.

Huse SM, Ye YZ, Zhou YJ, Fodor AA. (2012). A core human microbiome as viewed through 16S rRNA sequence clusters. PLoS One 7: e34242.

Ishii K, Mussmann M, MacGregor BJ, Amann R. (2004). An improved fluorescence in situ hybridization protocol for the identification of bacteria and archaea in marine sediments. FEMS Microbiol Ecol 50: 203-213.

Kikuchi Y, Graf J. (2007). Spatial and temporal population dynamics of a naturally occurring two-species microbial community inside the digestive tract of the medicinal leech. Appl Environ Microbiol 73: 19841991.

Koropatnick TA, Engle JT, Apicella MA, Stabb EV, Goldman WE, McFall-Ngai MJ. (2004). Microbial factormediated development in a host-bacterial mutualism. Science 306: 1186-1188.
Lane DJ. (1991). 16S/23S rRNA sequencing. In Stackebrandt E, Goodfellow M (eds). Nucleic Acid Techniques in Bacterial Systematics. John Wiley and Sons: New York, NY, USA, pp 115-175.

Ludwig W, Strunk O, Westram R, Richter L, Meier H, Yadhukumar et al. (2004). ARB: a software environment for sequence data. Nucleic Acids Res 32: 1363-1371.

Manz W, Amann R, Ludwig W, Vancanneyt M, Schleifer KH. (1996). Application of a suite of $16 \mathrm{~S}$ rRNAspecific oligonucleotide probes designed to investigate bacteria of the phylum cytophaga-flavobacterbacteroides in the natural environment. Microbiology 142 (Pt 5): 1097-1106.

Nyholm SV, Graf J. (2012). Knowing your friends: invertebrate innate immunity fosters beneficial bacterial symbioses. Nat Rev Microbiol 10: 815-827.

Parrinello N, Arizza V, Cammarata M, Giaramita FT, Pergolizzi M, Vazzana $M$ et al. (2007). Inducible lectins with galectin properties and human IL1alpha epitopes opsonize yeast during the inflammatory response of the ascidian Ciona intestinalis. Cell Tissue Res 329: 379-390.

Parrinello N, Patricolo E, Canicatti C. (1977). Tunicate immunobiology. I. Tunic reaction of Ciona intestinalis L. to erythrocyte injection. Bolletino di zoologia 44: 373-381.

Pernthaler A, Pernthaler J, Amann R. (2002). Fluorescence in situ hybridization and catalyzed reporter deposition for the identification of marine bacteria. Appl Environ Microbiol 68: 3094-3101.

Pichon D, Gaia V, Norman MD, Boucher-Rodoni R. (2005). Phylogenetic diversity of epibiotic bacteria in the accessory nidamental glands of squids (Cephalopoda: Loliginidae and Idiosepiidae). Marine Biology 147: 1323-1332.

Pinto MR, Chinnici CM, Kimura Y, Melillo D, Marino R, Spruce LA et al. (2003). CiC3-1a-mediated chemotaxis in the deuterostome invertebrate Ciona intestinalis (Urochordata). J Immunol 171: 5521-5528.

Pruesse E, Peplies J, Glockner FO. (2012). SINA: accurate high-throughput multiple sequence alignment of ribosomal RNA genes. Bioinformatics 28: 1823-1829.

Quince C, Lanzen A, Davenport RJ, Turnbaugh PJ. (2011). Removing noise from pyrosequenced amplicons. $B M C$ Bioinformatics 12: 38 .

Rath CM, Janto B, Earl J, Ahmed A, Hu FZ, Hiller L et al. (2011). Meta-omic characterization of the marine invertebrate microbial consortium that produces the chemotherapeutic natural product ET-743. ACS Chem Biol 6: 1244-1256.

Riesenfeld CS, Murray AE, Baker BJ. (2008). Characterization of the microbial community and polyketide biosynthetic potential in the palmerolide-producing tunicate Synoicum adareanum. J Nat Prod 71: 1812-1818.

Sasaki N, Ogasawara M, Sekiguchi T, Kusumoto S, Satake H. (2009). Toll-like receptors of the ascidian Ciona intestinalis: prototypes with hybrid functionalities of vertebrate Toll-like receptors. J Biol Chem 284: 27336-27343.

Schloss PD, Westcott SL, Ryabin T, Hall JR, Hartmann M, Hollister EB et al. (2009). Introducing mothur: open-source, platform-independent, community-supported software for describing and comparing microbial communities. Appl Environ Microbiol 75: 7537-7541. 
Schloss PD, Gevers D, Westcott SL. (2011). Reducing the effects of PCR amplification and sequencing artifacts on 16S rRNA-based studies. PLoS One 6: e27310.

Schmidt EW, Donia MS. (2010). Life in cellulose houses: symbiotic bacterial biosynthesis of ascidian drugs and drug leads. Curr Opin Biotechnol 21: 827-833.

Schmidt EW, Nelson JT, Rasko DA, Sudek S, Eisen JA, Haygood MG et al. (2005). Patellamide A and C biosynthesis by a microcin-like pathway in Prochloron didemni, the cyanobacterial symbiont of Lissoclinum patella. Proc Natl Acad Sci USA 102: 7315-7320.

Shade A, Hogan CS, Klimowicz AK, Linske M, McManus PS, Handelsman J. (2012). Culturing captures members of the soil rare biosphere. Environ Microbiol 14: 2247-2252.

Small KS, Brudno M, Hill MM, Sidow A. (2007). A haplome alignment and reference sequence of the highly polymorphic Ciona savignyi genome. Genome Biol 8: R41.

Turnbaugh PJ, Hamady M, Yatsunenko T, Cantarel BL, Duncan A, Ley RE et al. (2009). A core gut microbiome in obese and lean twins. Nature 457: 480-484.

Wang Q, Garrity GM, Tiedje JM, Cole JR. (2007). Naive Bayesian classifier for rapid assignment of rRNA sequences into the new bacterial taxonomy. Appl Environ Microbiol 73: 5261-5267.

Supplementary Information accompanies this paper on The ISME Journal website (http://www.nature.com/ismej) 